\title{
Expectations structure in asset pricing experiments
}

\author{
Giulio Bottazzi ${ }^{1}$ and Giovanna Devetag ${ }^{2}$ \\ 1 Sant'Anna School of Advanced Studies, Piazza Martiri della Libertà, 33 - \\ I-56127 Pisa, Italy bottazzi@sssup.it. \\ 2 Department of Management and Computer Science, University of Trento, Via \\ Inama, 5 - 38100 Trento, Italy devetag@cs.unitn.it
}

Summary. Notwithstanding the recognized importance of traders' expectations in characterizing the observed market dynamics, for instance the formation of speculative bubbles and crashes on financial markets, little attention has been devoted so far by economists to a rigorous study of expectation formation in the laboratory.

In this work we describe a laboratory experiment on the emergence and coordination of expectations in a pure exchange framework. We largely base our study on previous experiments on expectation formation in a controlled laboratory environment by Cars Hommes, Joep Sonnemans, Ian Tuinstra and Henk van de Velden (2002a).

We consider a simple two asset economy with a riskless bond and a risky stock. Each market is composed of six experimental subjects who act as financial advisors of myopic risk-averse utility maximizing investors and are rewarded according to how well their forecasts perform in the market. The participants are asked to predict not only the price of the risky asset at time $t+1$, as in Hommes et al. (2002a), but also the confidence interval of their prediction, knowing the past realizations of the price until time $t-1$. The realized asset price is derived from a Walrasian market equilibrium equation, unknown to the subjects, with feedback from individual forecasts. Subjects' earnings are proportional to the increase in their wealth level. With respect to previous experiments that did not include an explicit evaluation of risk by participants, we observe a higher price volatility, a decreased likelihood of bubble dynamics and, in general, a higher heterogeneity of predictions.

Key words: experimental economics, expectations, coordination, asset pricing

JEL codes: C91,C92,D84,G12,G14

\section{Introduction}

It is by now largely acknowledged that investors' expectations play a key role in the formation of speculative bubbles and crashes on financial markets. However, notwithstanding the recognized importance of trying to asses 
the dynamics that govern the formation of expectations and their response to information and experience, little attention has been devoted so far by economists to a rigorous study of expectation formation in the laboratory.

There are several experimental studies on asset markets (see, among others, [4], [5], [6], [7], [8]. However, most of these studies' main focus is the process of trading and the way in which trading activity itself may push prices far above or below the asset's fundamental value, although in several cases information about the participants' expectations is collected as well (see [9] for a review).

In this work we describe a simple laboratory experiment on the emergence and coordination of expectations in a financial market, in which no trading takes place and in which subjects' only task is to form predictions about the future return of an asset and give a confidence range of their prediction. We largely base our study on a previous experiment of this type by [2] on expectation formation in a controlled laboratory environment. In their experiment, each market is composed of six experimental subjects who are asked to predict the price of a risky asset at time $t+1$ knowing the past realizations of the price until time t-1, the mean dividend at each period and the riskfree interest rate. Subjects' earnings are a function of their forecasting errors, and the realized asset price is derived from an unknown market equilibrium equation with feedback from individual forecasts. The authors find out that in most markets prices diverge significantly from the (constant) fundamental value; moreover, speculative bubbles emerge endogenously due to positive feedback expectations. In a related experiment ([3]), the authors introduce a small fraction of artificial, fundamentalist traders. In this latter case, the market dynamics show regular oscillations of the price around the fundamental value, and excess volatility. In both experiments, subjects belonging to the same market tend to coordinate on a common prediction strategy.

We replicate the experimental design in [2], but changing some important features. First and most importantly, we ask our subjects to predict not only the future price of the asset, but also the confidence interval of their prediction, which we use as an estimate of the forecasted variance of the returns. In this way subjects are asked to provide an estimation of the risk associated with their prediction and we are recreating a framework that is more similar to the ones really faced by financial advisors. We believe that adding information about the forecasted variance in the model may affect the dynamics observed in the experimental market compared to the baseline case of $[2,3]$.

Secondly, in our experiments a subject's earnings do not depend on his forecasting errors, but are proportional to the increase in the wealth level of a hypothetical investor (played by the computer program) who makes trading decisions according to a mean-variance approach taking as inputs the subjects' forecasts and acting with the time horizon of one step. In other words, experimental subjects act as financial advisors of myopic risk-averse utility maximizing investors and are rewarded according to how well their forecasts perform in the market. This experimental design allows us to focus exclusively 
on the dynamics of subjects' expectations, as in the original experiment by Hommes et al; in fact, our subjects are not engaged in any trading, which is done automatically by the computer program on the basis of the predictions they provide; however, the payoff assignment rule that we adopt is more correspondent to what happens in real financial markets, where prediction accuracy only matters inasmuch as it leads to 'correct' trading decisions.

Finally, we inform subjects of the positive feedback mechanism governing the dynamics of price. In particular, subjects know that the price at time $t$ is an increasing function of all market participants' forecasted returns at time $t+1$, and a decreasing function of the forecasted variability of the predicted returns. Subjects are also informed about the way in which the computer program calculates their personal demand functions for shares on the basis of their predictions, and on the nature of the asset pricing equation. In other words, subjects know that the price level at each time step - and the corresponding return - is generated by their own forecasting activity in a deterministic way. With such additional information, there is the possibility for groups to coordinate on a common expectation strategy. Section 2.1 and Section 3 respectively describe our model of asset pricing and our experimental design and implementation. Section 4 discusses the results and Section 5 offers some concluding remarks.

\section{The Model}

As previously said, participants in our experiment are asked to act as financial advisors for mean-variance utility maximizer speculators with a short (one time step) time horizon. We consider a simple economy with one risky stock paying a constant dividend $D$ at each trading round, and a riskless bond with a constant return $R$. At each round of the experiment the participants are asked to provide a prediction of the risky asset price return in the next round, i.e., of the relative price increase between the present and the next round, together with a confidence interval for their prediction. The participants' predictions are then used as inputs to drive the investment decisions of synthetic risk averse traders, played by the computer, which finally determine the asset price for that round. In this section we describe the behavior of the synthetic traders in order to derive the explicit rules by which the participants' predictions do actually generate the price at every time step. We begin by describing the traders' demand functions following [1] and, afterwards, describe how the individual demand functions are merged to obtain the asset pricing equation.

\subsection{Personal demand function}

Let $W(t)$ be the trader wealth at the beginning of time $t$ and let $x$ be the fraction of wealth he wants to invest in the risky asset. If the price of the asset is $p(t)$ and $A(t)$ is the number of possessed shares then $W(t) x=p(t) A(t)$. 
The future trader wealth (i.e. its wealth at the beginning of the next round) depends on the future return on the stock price $h(t)=p(t+1) / p(t)-1$ and reads

$$
W(t+1 ; h(t))=x W(t)(h(t)-R+D / p(t))+W(t)(1+R)
$$

where the dividends $D$ are paid after the payment of the riskless interest $R$ at the end of round $t$.

We choose as the expression of the agent utility the simplest function of the expected return and variance

$$
U(t)=E_{t-1}[W(t+1)]-\frac{\beta}{2} V_{t-1}[W(t+1)]
$$

where $E_{t-1}[$.$] and V_{t-1}[$.$] stand respectively for the expected return and vari-$ ance computed at the beginning of round $t$, i.e. with the information available at time $t-1$, and where $\beta$ is the "risk-aversion" parameter.

Using the expression for $W$ in (1) one obtains

$$
E_{t-1}[W(t+1)]=x W(t)\left(E_{t-1}[h(t)]-R+D / p(t)\right)+W(t)(1+R)
$$

and

$$
V_{t-1}[W(t+1)]=x^{2} W(t)^{2} V_{t-1}[h(t)] .
$$

Substituting (3) and (4) in (2) one obtains

$U(t)=x W(t)\left(E_{t-1}[h(t)]-R+D / p(t)\right)-x^{2} \frac{\beta}{2} W(t)^{2} V_{t-1}[h(t)]+W(t)(1+R)$

whose maximum, remembering the definition of $x$ provides the agent's demand of asset at times $t, A(t)$ that reads

$$
A(t)=\frac{E_{t-1}[h(t)]-R+D / p(t)}{\beta V_{t-1}[h(t)] p(t)}
$$

\subsection{Aggregate demand and pricing equation}

Consider a population of $N$ heterogeneous traders. Let $\beta_{i}$ be (with $i \in$ $\{1, \ldots, N\})$ the risk aversion of the $i$-th trader and $E_{t-1, i}$ and $V_{t-1, i}$ respectively his forecasted return and variance. If the total amount of risky asset is $A_{\text {tot }}$ its price can be determined under the condition of reducing the excess demand to zero. One has that the price $p(t)$ must solve

$$
\sum_{i=1}^{N} \frac{E_{t-1, i}-R+D / p(t)}{\beta_{i} V_{t-1, i} p(t)}=A_{\text {tot }}
$$

that reduces to a second order equation whose positive root reads 


$$
p(t)=\frac{\bar{E}_{t-1}}{2}+\sqrt{\left(\frac{\bar{E}_{t-1}}{2}\right)^{2}+\bar{D}_{t-1}}
$$

where

$$
\bar{E}_{t-1}=\sum_{i=1}^{N} \frac{E_{t-1, i}-R}{A_{\mathrm{tot}} \beta_{i} V_{t-1, i}}
$$

is an average expected excess return weighted with respect to the inverse risk and

$$
\bar{D}_{t-1}=\sum_{i=1}^{N} \frac{D}{A_{\mathrm{tot}} \beta_{i} V_{t-1, i}}
$$

is the analogously weighted expected dividend.

Note that (8) provide a positive price for the risky asset even if the average expected excess return $\bar{E}_{t-1}$ is negative. Note that the above pricing equation has been obtained without considering any budget constraint for the agents. The $A_{\text {tot }}$ can be reabsorbed in an overall rescaling of the parameters $\beta_{i}$.

\subsection{The skeleton of the experiment}

We conducted experiments with a given number of subjects acting as advisors for identical traders, in particular having an equal degree of risk aversion $\beta$. The experiment structure can be roughly described as follows:

- each participant is asked to provide a range within which she predicts, with a high degree of probability ${ }^{3}$, the future return will lay. Let $a_{i}, b_{i}$ the range provided by participant $i \in\{1, \ldots, N\}$.

- for each participant range we compute the mid value and take it as an estimate of its forecasted return $E_{i}=\left(b_{i}+a_{i}\right) / 2$. The forecasted variance of player $i$ is set to $V_{i}=\left(b_{i}-a_{i}\right)^{2} / 4$, i.e. the provided range is put equal to the $2 \sigma$ range of an (assumed) underlying normal distribution.

- (8) is used to fix the present price $p(t)$, then (6) is used to determine the present market position of the synthetic trader, implied by the predictions of $i$-th participant, $A_{i}(t)$.

- once price at time $t$ is determined, from the trader's position in the previous time step $A_{i}(t-1)$ and from the past price $p(t-1)$ it is possible to determine the present value of her past position. The profit earned by the prediction of the $i$-th participant reads:

$$
\pi_{i}(t)=A_{i}(t-1)(p(t)+D-p(t-1)(1+R))
$$

Notice that the total number of assets and the synthetic traders' degree of risk aversion (as measured by the parameter $\beta$ ) can be used to tune the

\footnotetext{
${ }^{3}$ Roughly $95 \%$, equivalent to the 2 standard deviation range of a normal distribution
} 
impact of the forecasted variance on the price of the asset. At the end of the round $t$ each participant receives information about: the new price $p(t)$, the new return $h(t)$, and the realized profit from the previous round $\pi_{t}$. In the next Section a detailed description of the experimental design is provided.

\section{The experimental design and implementation}

The experiment was entirely computerized and it took place in one of the computer rooms of the Computable and Experimental Economics Lab of the University of Trento on May 7, 2003. We conducted two sessions of the experiment, with three cohorts of 6 subjects each participating in the first session and two cohorts of six participants in the second session, for a total of 30 subjects, who were recruited through ads posted at the various department buildings.

Subjects were mostly undergraduate students in economics and had never participated in experiments of this type before. Before the experiment began, subjects received paper copies of the instructions which were read aloud by the experimenter to make sure that the rules of the market were common knowledge among participants ${ }^{4}$. In addition, a handout with a summary of the market functioning together with the specification of the relevant parameters was also given to every subject. All subjects could see each other but were prevented from looking at each other's computer screens by wooden separators. They were told that they would participate in a financial market composed of six participants in which their task was to predict the expected price return of a hypothetical asset and its range of variability for a total of 50 periods. Their earnings would depend on the increase in the value of an initial endowment $\mathrm{X}$ that they would have at the beginning of every period. Instructions explained to subjects how their given forecasts of the expected price return at time $t+1$ $E_{i}(t+1)$ and the confidence interval $V_{i}(t+1)$ would be utilized by the software to compute their personal demand function in every round, and how the sum of the personal demand functions of all six participants would determine the market price of the asset in period $t$. It was explained to subjects that the range $V$ would be the interval of values that according to them would contain the forecasted return with a probability roughly equal to $95 \%$.

Finally, instructions also explained that their earnings at the end of each period would consist of the capital gain achieved (which of course depended on the realized price return), the dividends paid and the fixed interest rate $R$ gained on the residual of their endowment. It was made clear that there was no dependence between the investment choices made in different rounds, as a new, initial endowment $\mathrm{X}$ would be available to them at the beginning of every period. In other words, subjects acted as financial advisors of different investors, each of which acted as a mean-variance optimizer with a time horizon of one step.

\footnotetext{
${ }^{4}$ A complete English translation of the instructions is available upon request.
} 

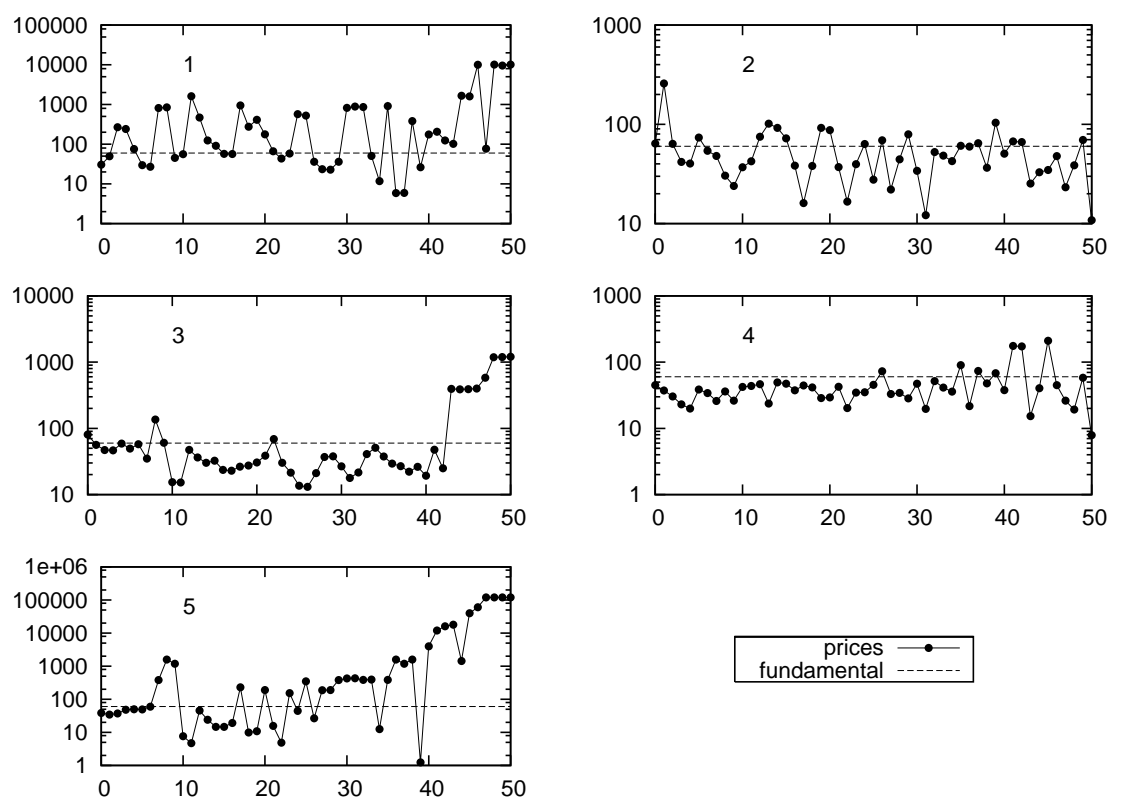

Fig. 1. Price history for the 5 groups

Subjects were told that their demand of shares could be negative or zero in some rounds, meaning that they could hold short positions or invest all of their endowment in the acquisition of bonds that would yield the riskless rate $R$. Their final earnings would be determined by the sum of their earnings in every round, and converted into cash as follows: the participant within the group that had achieved the highest cumulative payoff would receive an amount of $25 €$, while the participant with the minimum cumulative payoff would receive an amount of $5 €$. All other would get a payoff proportional to the maximum achieved. Subjects were informed of the payoff assignment rule but did not have any information throughout the experiment about other participants' earnings, therefore such payoff assignment rule gave them the highest incentive to maximize their earnings. Finally, although subjects knew that the market would be composed of six participants, they did not know the identity of the other five participants.

Sessions lasted one hour and a half each. The initial endowment $X$ was set equal to 100 experimental schillings, the risk-less interest rate $R$ was equal to $5 \%$, the dividend was equal to 3 schillings. The parameters $\beta$ and $A_{\text {tot }}$ were fixed to the values of 100 and 1 , respectively. 

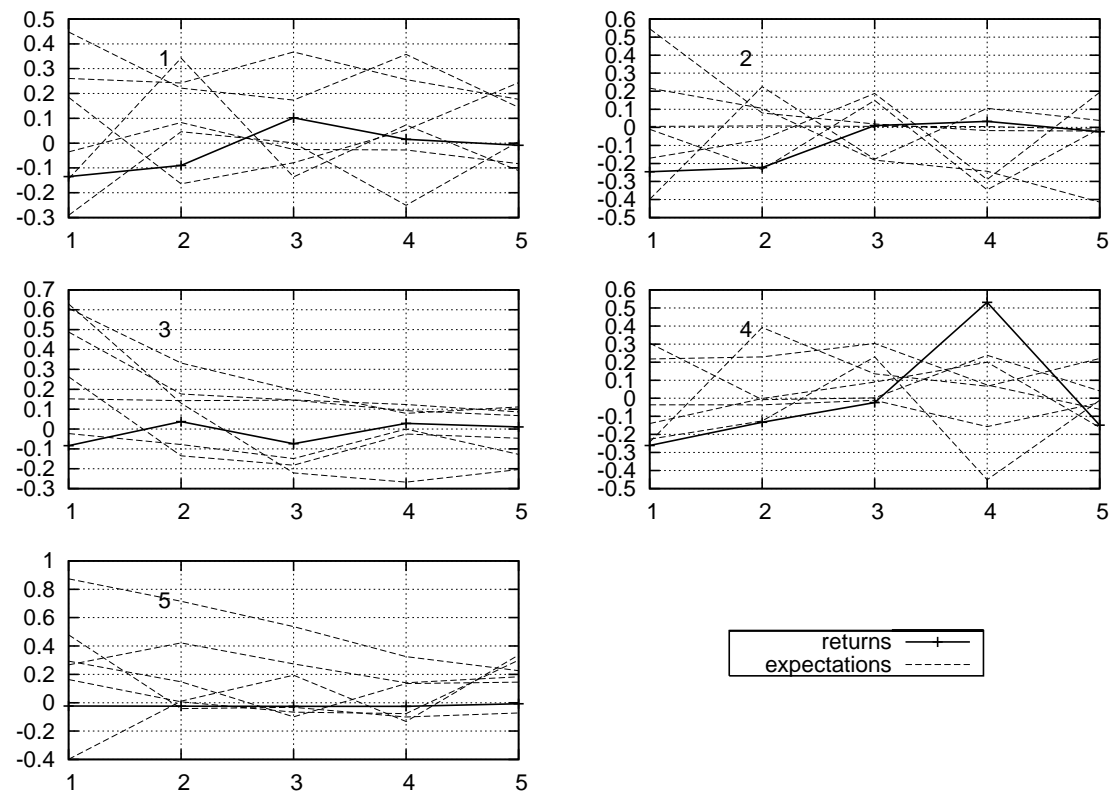

Fig. 2. Autocorrelogram of price returns for the 5 groups

\section{Results}

In Fig. 1 we report the observed asset prices $p(t)$ for the 5 different groups together with the asset fundamental value $\bar{p}=60$. As can be seen from the plots, price volatility is very high but, except for the final rounds of groups 3 and 5 , it oscillates around the fundamental value. Moreover, notice that the price increase observed in group 3 and 5 is inconsistent with an idea of rational bubble, since it does not display the expected exponential growth. Hence, these can be plausibly interpreted as "speculative" bubbles. Their appearance toward the end of the experiment is probably due to a more 'extremist' behavior of some of the participants as the end of the experiment is approaching.

The behavior of the asset price in our experiment is remarkably different from the one observed in $[2,3]$, exhibiting a higher volatility and a substantial lack of creation of relatively stable bubbles.

In Fig. 2 the autocorrelation structure of asset price returns $r(t)=$ $p(t+1) / p(t)-1$ for the 5 groups is shown (bold line) together with the autocorrelation of expected returns $E_{t, i}$. The former is analogous in all groups except group 5, showing a tendency toward anti-correlation at 1 time lag. As far as the forecasted returns are concerned, one can notice that their autocorrelation structure is rather heterogeneous, with, however, a general tendency toward positive correlation, in contrast with the observed structure in price returns. 

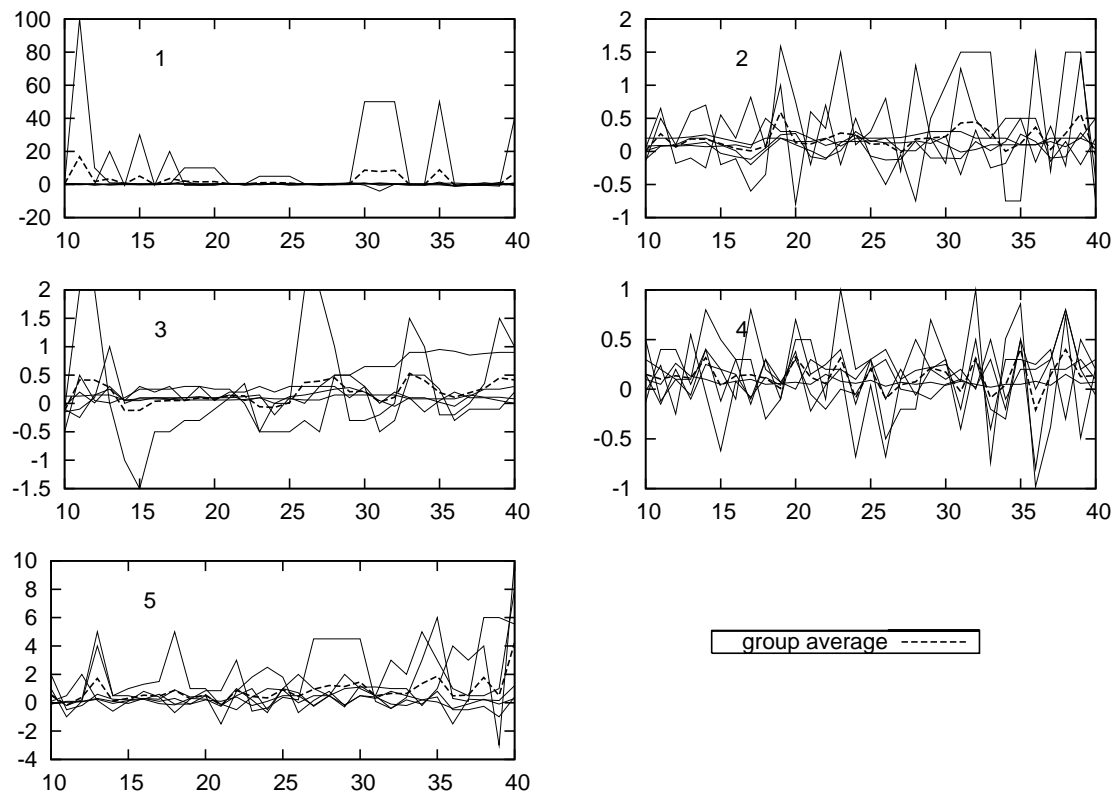

group average --------

Fig. 3. Forecasted returns for the 5 groups. Each line corresponds to the forecast of one agent

Table 1. Average and standard deviation of players forecasted returns computed over 10 rounds

\begin{tabular}{lccccc}
\hline time & group 1 & group 2 & group 3 & group 4 & goup 5 \\
\hline 10 & $4.061(15.688)$ & $0.129(0.217)$ & $0.326(1.336)$ & $0.117(0.238)$ & $0.510(1.100)$ \\
20 & $1.617(5.011)$ & $0.173(0.402)$ & $0.029(0.312)$ & $0.157(0.275)$ & $0.427(0.884)$ \\
30 & $2.812(10.972)$ & $0.224(0.449)$ & $0.224(0.490)$ & $0.074(0.306)$ & $0.827(1.239)$ \\
40 & $1.585(8.221)$ & $0.189(0.511)$ & $0.285(0.408)$ & $0.132(0.425)$ & $2.838(8.731)$
\end{tabular}

The heterogeneity in the participants' forecasts can be better judged from Fig. 3 and Fig. 4 that report the forecasted returns and forecasted variances of all the participants. We have eliminated the very beginning and end of the experiment in which some of the participants exhibit strange behaviors, probably due to lack of learning in the former case and end-of-experiment effects in the latter. As can be seen, not only predictions are quite heterogeneous, but they do no display any tendency to converge.

In Table 1 and Table 2, we also report the average and standard deviation values, calculated over successive 10 rounds of trading, of the agents' forecasted returns and variances, respectively. As can be seen, the standard deviation of both the predictors, reported in parenthesis, does not decrease during the experiment in any of the 5 groups. The volatility level is groupspecific, but it is always very high and stationary. Forecasted variances seem 

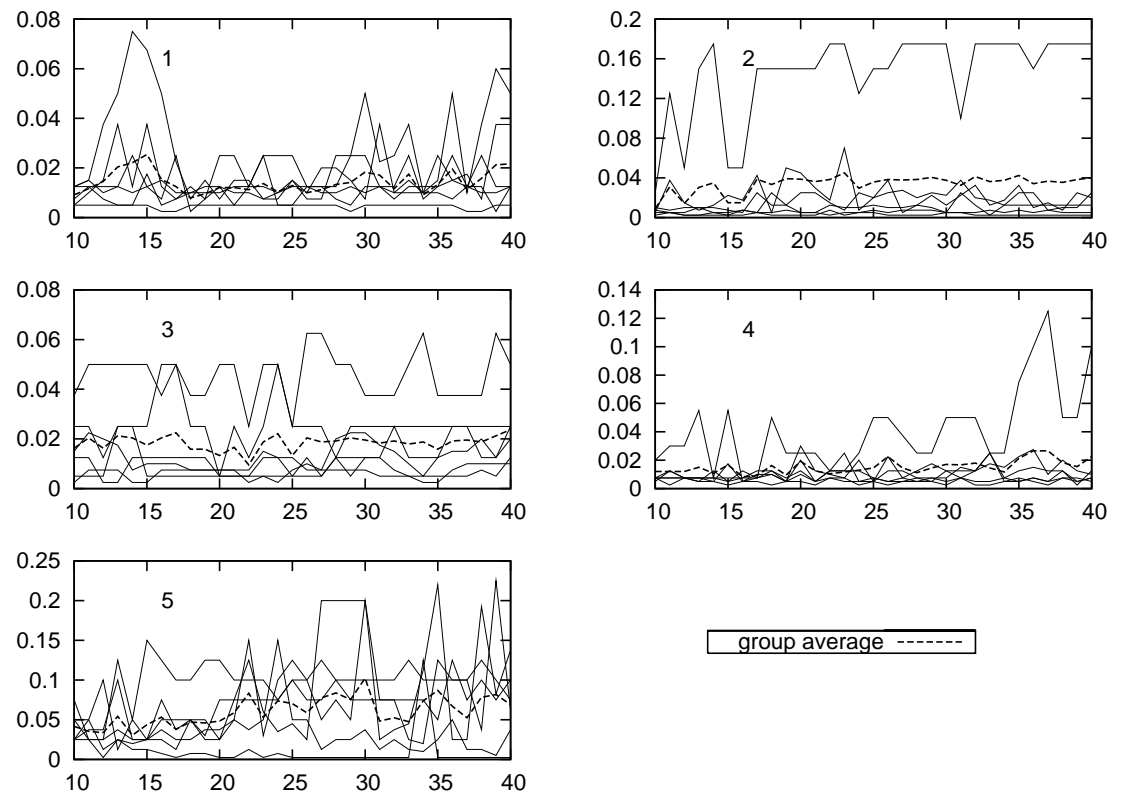

Fig. 4. Forecasted variances for the 5 groups. Each line corresponds to the forecast of one agent

Table 2. Average and standard deviation (inside parenthesis) of players forecasted variances computed over 10 rounds

\begin{tabular}{lccccc}
\hline time & group 1 & group 2 & group 3 & group 4 & goup 5 \\
\hline 10 & $0.012(0.008)$ & $0.016(0.027)$ & $0.016(0.014)$ & $0.012(0.011)$ & $0.029(0.031)$ \\
20 & $0.014(0.014)$ & $0.033(0.052)$ & $0.017(0.016)$ & $0.012(0.010)$ & $0.050(0.039)$ \\
30 & $0.014(0.009)$ & $0.037(0.056)$ & $0.019(0.016)$ & $0.016(0.014)$ & $0.069(0.056)$ \\
40 & $0.018(0.019)$ & $0.038(0.061)$ & $0.020(0.016)$ & $0.021(0.028)$ & $0.077(0.068)$ \\
\hline
\end{tabular}

to exhibit a higher persistence, in contrast to the forecasted returns. The more risk averse participants can be clearly perceived in Fig. 4, and seem to behave consistently over time.

Finally, in Fig. 5 we report the lagged cross correlation between the agents' forecasted returns and the realized price returns $\left\langle r_{t}, E_{t+\tau, i}\right\rangle$ for $\tau \in[-3, \ldots, 3]$. In this way one can analyze both how participants take the past returns into account when formulating their predictions, and how the participants' predictions themselves impact on the asset price. As expected, there is a strong correlation at lag 0. A high prediction of tomorrow's returns formulated today generates a price increase and, consequently, a positive realized return today. Interestingly, the correlation between today's forecasts and tomorrow's prices is negative, suggesting a tendency to over-react to market fluctuations. This is plausibly the reason of the observed 1 lag negative 

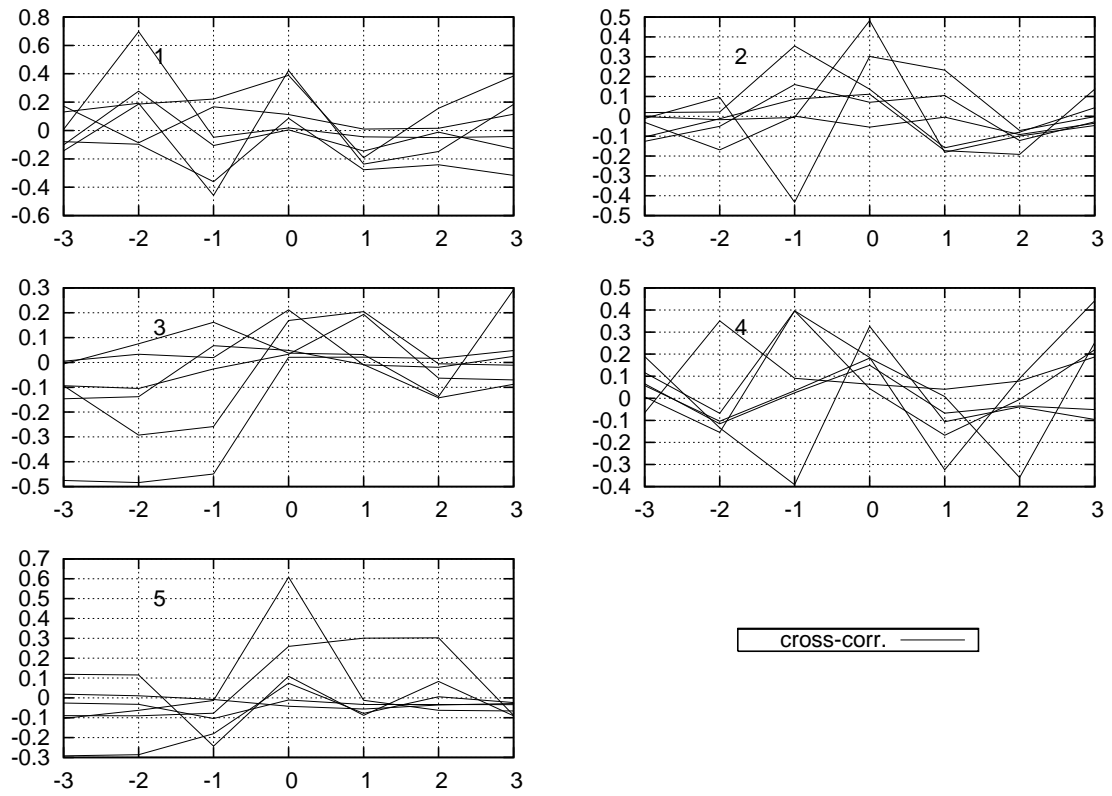

Fig. 5. Cross-correlogram between agents forecasted returns and realized returns

autocorrelation in Fig. 2, i.e., of price "bouncing" dynamics. The correlation structure between the participants' predicted returns and past price returns is extremely heterogeneous and only a low degree of persistence can be observed in some cases.

\section{Conclusions}

In this paper we have described a simple asset pricing experiment in which participants must forecast both the expected future price return of an asset and its variance. Our results show that, with respect to previous experiments in which subjects had to predict only the future price value, - and the pricing equation was linear with positive feedbacks from individual predictions - we find no evidence of coordination on common expectations, no evidence of persistent speculative bubbles, and a high volatility in the realized price around the asset's fundamental value. In [3], the introduction of fundamentalist traders prevented the formation of speculative bubbles but did not prevent traders' coordination on a common prediction strategy. This coordination is not observed in our experimental markets. We suggest that it is the nonlinear structure of our pricing equation to determine such dynamics; in fact, the inclusion of the forecasted variance determines a dynamic by which small changes in the predicted confidence range determine large fluctuations in the 
realized price, which in turn makes it very difficult for subjects to coordinate on a common strategy. Note that in our model we adopted the simplifying assumption of an equal degree of risk aversion for our subjects. Future experiments should aim at controlling for the - presumably - different risk attitudes of the participants in the experimental market, which are a relevant determinant of predictions and expectations in this type of settings. Another possible contribution would be to induce different risk attitudes to the subjects and test for its effect on coordination. Taken together, our results confirm the importance of the market institutional details - and in particular the nature of the expectations feedback structure - in determining the observed market dynamics.

\section{Acknowledgments}

The authors thank participants in WEHIA 2003 and an anonymous referee for useful comments and suggestions, Marco Tecilla and Ivan Soraperra for software development. The first author acknowledges financial support from the St.Anna School of Advanced Studies (grant n. E6003GB). The second author acknowledges support from MIUR (Cofin 2002). The usual disclaimer applies.

\section{APPENDIX}

\section{A Instructions}

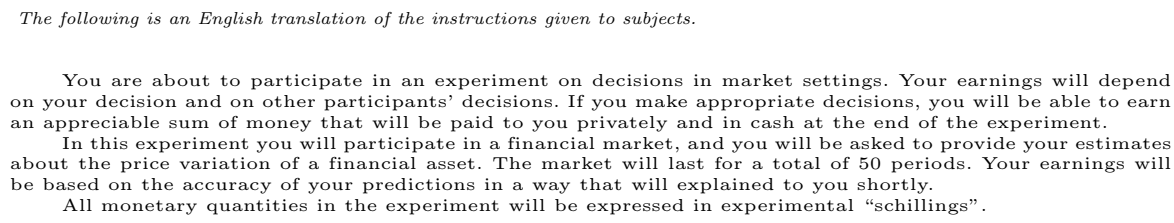

\section{The Market}

Each of you will participate in a market in which a hypothetical asset will be traded. Each market is composed of six participants (hence, yourself and other five participants). The division between groups of six participants will be made by the software randomly at the beginning of the experiment, and the group composition will remain the same throughout the experiment. The only information that you will have about the market will be the price of the asset over time, and its corresponding return (i.e., the price variation from one period to the next). Thi financial asset is such that at the end of every period a fixed dividend of 3 schillings is paid for each share owned (the dividends, for those of you who don't know it, represent the distribution of a firm's profits to the by the by the computer program in the following way. 


\section{Predictions}

At the beginning of period $t$ - hence having information only up to period $t-1$ - you will be asked to provide two estimates:

1. your estimate of the asset's price variation in period $t+1$, or, in other words, its return, which we call $E$. This value can be positive or negative

2. Your confidence range for such estimate, i.e., the range in which, according to you, the actual return $E$ will fall with a probability roughly equal to $95 \%$. We will label this confidence range with $V$

For example, you may predict that the asset return will be equal to $5 \%$, with a confidence range of $\pm 1 \%$. . $4 \%$ to a maximum of $6 \%$ with a probability of roughly $95 \%$.

The return is simply the price variation from round to round, defined as follows:

$$
\frac{\operatorname{price}(t+1)-\operatorname{price}(t)}{\operatorname{price}(t)} \times 100
$$

Clearly, if the price rises the return will be positive, whereas if the price falls, the corresponding return will be negative.

\section{Investment}

At the beginning of each period you have an endowment of 100 schillings, given to you by a hypothetical bank, and which you can invest entirely or in part in purchasing shares of the asset. The amount that you do not use to buy shares remains in the bank and gives you a fixed (riskless) return of $5 \%$ in each period. On the basis of your estimates of the return of the asset at time $t+1$ and on the confidence range of your estimates (i.e., the two values of $E(t+1)$ and $V(t+1))$, the software will compute the number of shares that you purchase at th beginning of period $t$ according to the following equation:

$$
D(t)=\frac{E(t+1)-0.05+3 / p(t)}{[V(t+1) / 2]^{2} p(t)}
$$

where $E(t+1)$ and $V(t+1)$ are your forecasted return and its confidence range respectively, 0.05 is the riskless rate of interest that the bank applies to you, 3 is the dividend paid by each share in each period. In other words, $D(t)$ is your individual demand of shares that the software calculates automatically for you in every period. The higher your estimated return for the next period $(E(t+1))$ the higher your demand for shares in this period. On the contrary, the higher your confidence range (which reflects your degree of uncertainty about your own prediction of the future return), the lower will be your demand for shares. Please note that the variable $p(t)$, which represents the price of the asset in period $t$, is an unknown in the equation, and it will be determined as follows.

\section{Equilibrium between demand and supply and determination of the asset price}

The price of the asset at time $\mathrm{t}$ is determined by equating the total demand for shares to the total supply of shares:

$$
\sum_{i=1}^{6} \frac{E_{i}(t+1)-0.05+3 / p(t)}{\left[V(t+1)_{i} / 2\right]^{2} p(t)}=X \rightarrow p(t)
$$

the term on the left-hand side of the equation is simply the sum of the individual demands of shares of all six participants, while $X$ is the total supply of shares, which remains fixed throughout the experiment. Since the supply of shares is tixed, the higher the market demand for shares, the higher will be the price of the asset. The about such expectations (i.e. the lower the confidence rane). After determining the price of the asset in period $t$ the software will sult you will buy.

\section{Your Payoff}

We assume for simplicity that everything you buy at the beginning of a period is sold in the following period. Your earnings will depend on how you have invested the endowment of 100 schillings that you have at the beginning of each period, and may derive from three sources: the increment of value of the shares you own, the payment of that realized at the end of period $t+1$ and will be determined as follows:

$$
\pi(t+1)=D(t)(p(t+1)-p(t))+3 D(t)+0.05 \times(100-D(t) p(t))
$$


The first term expresses your capital gain, i.e., the profit you earn by reselling at price $(t+1)$ the shares that you bought at price $(t)$; the second term is the earning you get from payment of dividends ( 3 shillings for each share), and the third term is the riskless interest gained on the residual. Note that you are allowed to borrow money to buy shares or sell shares that you don't own in order to 'buy' money. Hence, $D(t)$ may be negative in one period (in which case you will sell shares instead of buying them and will buy them back at the end of the subsequent period; you will earn a capital gain if the price has decreased); or, the value of $D(t) p(t)$ may be he negative, reflecting the interes a period, your earnings will be given simply by the $5 \%$ interest on your entire endowment. In brief, your payoff will depend on the choices of investment that the software will make for you on the basis of your predictions. Important: at the beginning of the next period, you again have a new endowment of 100 schillings. Everythin you bought is resold before a new period starts. Therefore, there is no dependence between your investment plans in different periods.

\section{A Numerical Example}

At the beginning of period 4 - and knowing the asset price up to period 3 -you have to make a prediction on the asset return (and its confidence range) in period 5. Let's us assume that you predict a return of $12 \%$ with a confidence interval of $\pm 5 \%$. The software computes the optimal quantity of shares that you buy in period 4 , as a function of price in period 4, which is still unknown. When all participants have entered their predictions, the individual demand for shares will be summed up and put equal to the supply of shares, which is constant. Solving the equation (demand=supply) will determine the asset price in period 4, which we assume to be equal to 10.2. Now, the software substitutes the value of 10.2 to $p(4)$ in your individual demand equation to find the number of shares that you buy. Let's us assume this number is 5; therefore, in period 4 you buy shares for a total value of $5 * 10.2=51$ schillings. At the end of period 5, you can see the earnings that you obtained with thi investment in period 4 . Let's assume that the price at time 5 is 12.8 , which is the price at which you will resell the shares. The corresponding return hence is equal to $(12.8-10.2) / 10.2=25 \%$. Your earnings will be equal to:

$$
G=5 \times(12.8-10.2)+(100-51) \times 0,05=13+2,45=15,45
$$

Now let's assume instead that the asset price has decreased in period 5 to value of 2 ; the corresponding return will be negative and equal to $(2-10.2) / 10.2=-80 \%$. You had mistakenly predicted a positive return and hence you had bought shares in period 4 . Your earnings in this case are:

$$
G=5 \times[2-10,2]+[100-(51)] \times 0,05=-41+2,45=-38,55
$$

A wrong prediction not necessarily means the you will suffer a loss; however, generally, the more accurate your prediction, the closer to optimal your investments will be, and higher your overall earnings. Your incentive is to provide predictions as accurate as possible. The confidence range reflects your degree of uncertainty on you own prediction; if you choose a wide confidence range, this implies you are very uncertain about the true value of the return, and hence you will buy a lowe rumer shares compared to the case in which you indicate a smaller confidence range. Your total earnings will be determined by the sum of the earnings you realize in each of the 50 perods and will be converted in eurs as follows: whoever anong the six participants to the market has achieved highest earning will receive $25 \mathrm{e}$. The participant with the lowest earnings will receive 5 e. All same rule will be applied in case you all suffer losses.

\section{Information}

The information that you will have in each period concerns the past realizations of the price, the actual return, your predicted return, your payoff in each round with the indication of your cumulated payoff between parentheses. Besides, the values or the price and the corresponding return will be also displayed graphically (by two market will las for 50 periods. At the berinning of a perion the vou see in the bottom part of the soreen by using the nut 'enter' The values you enter are already interpreted by the software as percentages, therefore you do not need to use decimal values. You can choose values between $-50,000$ and $+50,000$ for the return and you can choose values from 1 to 100 for the confidence range (you can simply insert a number for $\mathrm{V}$; for example, if you enter 2 , the software will automatically interpret it as $\pm 2 \%$. For the return, on the contrary, you also have to enter the sign $(+$ or -$)$.

Please, do the experiment in silence and do not try to look at other participants' screens. Are there any questions?

Thank you for your collaboration!

\section{References}

1. Bottazzi G. (2002) A Simple Micro-Model of Market Dynamics Part I: The "Homogeneous Agents" Deterministic Limit. L.E.M. Working Paper n. 200210, Pisa 
2. Hommes C, Sonnemans J, Tuinstra J and van de Velden H (2002) Expectations and Bubbles in Asset Pricing Experiments. CeNDEF Working Paper, Amsterdam

3. Hommes C, Sonnemans J, Tuinstra J and van de Velden H (2002) Coordination of Expectations in Asset Pricing Experiments. CeNDEF Working Paper, Amsterdam

4. King RR, Smith VL, Williams AW and van Boening M (1993) The Robustness of Bubbles and Crashes in Experimental Stock Markets. In: Prigogine I, Day $\mathrm{R}$ and Chen $\mathrm{P}$ (eds) Nonlinear Dynamics and Evolutionary Economics. Oxford University Press, Oxford

5. Noussair CN, Robin S and Ruffieux B (2001) Price Bubbles in Laboratory Asset Markets with Constant Fundamental Values. Experimental Economics 4:87-105

6. Porter DP, Smith VL (1995) Futures Contracting and Dividend Uncertainty in Experimental Asset Markets. Journal of Business 68:509-541

7. Smith VL, Suchanek GL and Williams AW (1988) Bubbles, Crashes, and Endogenous Expectations in Experimental Spot Asset Markets. Econometrica $56: 1119-1151$

8. Smith VL, van Boening M and Wellford CP (2000) Dividend Timing and Behavior in Laboratory Asset Markets. Economic Theory 16:567-583

9. Sunder S (1995) Experimental Asset Markets: A Survey. In: Kagel J and Roth A (eds) Handbook of Experimental Economics. Princeton University Press, Princeton, NJ 